\title{
EFEITO DE JOGOS DE RACIOCÍNIO LÓGICO SOBRE O SEDENTARISMO EM CRIANÇAS DE 7 A 9 ANOS NO COMBATE À OBESIDADE
}

\author{
Aranin Queiros de \\ Souza Santos ${ }^{1}$ \\ Mestranda em Ciências da \\ Saúde e Biológicas, \\ Universidade Federal do Vale do \\ São Francisco (UNIVASF). \\ E-mail: enf.aranin@gmail.com
}

\section{Ariane Queiroz de}

\section{Sousa ${ }^{2}$}

Mestranda em Ciências da

Saúde e Biológicas,

Universidade Federal do Vale do São Francisco (UNIVASF).

E-mail:

arianesousa2011@gmail.com

Luciana da Silva Lirani ${ }^{3}$ Doutora em Educação Física. E-mail: lucianalirani@gmail.com

\section{Ricardo Argenton} Ramos $^{4}$

Doutor em Ciência da Computação; Universidade Federal do Vale do São Francisco (UNIVASF). E-mail: ricargentonramos@gmail.com

\author{
THE EFFECTS OF LOGICAL REASONING GAMES ON \\ SEDENTARISM IN CHILDREN FROM 7 TO 9 YEARS OF AGE \\ IN COMBATTING OBESITY
}

\section{RESUMO}

A obesidade é considerada uma epidemia pela OMS e a sua incidência na população infantil é cada vez maior. Buscar soluções para mitigar o sedentarismo, uma das causas da obesidade infantil, tem sido o desafio de vários pesquisadores. Assim, o objetivo deste estudo foi avaliar os efeitos de jogos de raciocínio lógico sobre o sedentarismo em crianças de 7 a 9 anos no combate à obesidade. O estudo foi realizado em duas escolas públicas, no município de Petrolina-PE. A amostra foi composta por crianças (de ambos os sexos) do Ensino Fundamental I, faixa etária de 7 a 9 anos. Para mensurar a prática de atividade física foi aplicado o questionário Physical Activity Questionnaire for Older Children (PAQ-C). Na escola da intervenção as crianças participaram de oficinas, nas quais jogos de raciocínio lógico voltados ao combate da obesidade foram aplicados. Na escola controle as crianças foram igualmente avaliadas, porém não participaram de nenhuma atividade prática de jogos. Após a intervenção foi novamente reaplicado o questionário. Os resultados mostraram que o grupo caso se apresentou menos sedentário e mais moderadamente ativo após intervenção enquanto o grupo controle se mostrou mais sedentário. Infere-se que os jogos de raciocínio lógico no combate a obesidade infantil em crianças de 7 a 9 anos foram responsáveis em estimular o aumento dos níveis de atividade física e diminuir o sedentarismo.

PALAVRAS-CHAVE: Obesidade infantil. Jogos recreativos. Prevenção e controle 


\section{ABSTRACT}

Obesity is considered an epidemic by the WHO and its incidence in the child population is increasing. Finding solutions to alleviate sedentary lifestyle, one of the causes of childhood obesity, has been the challenge of many researchers. Thus, the objective of this study was to evaluate the effects of logic games on sedentarism in children aged 7 to 9 years in the fight against obesity. The study was carried out in two public schools, in the city of Petrolina-PE. The subjects consisted of children (of both sexes) of elementary school I, age group of 7 to 9 years. The Physical Activity Questionnaire for Older Children (PAQ-C) was used to measure physical activity. At the intervention school the children participated in workshops, in which logic games aimed at combating obesity were applied. In the control school the children were equally evaluated but, didn't participate in any practical games activity. After the intervention, the questionnaire was reapplied. The results showed that the case group presented less sedentary and more moderately active after intervention while the control group was more sedentary. It is inferred that logic games in the fight against childhood obesity in children aged 7 to 9 years were responsible for stimulating increased levels of physical activity and reducing physical inactivity.

PKEYWORDS: KIDSCREEN. Pediatric obesity. Games recreational. prevention e control.

\section{INTRODUÇÃO}

As doenças crônicas não-transmissíveis (DCNT) são caracterizadas por passarem longos períodos assintomáticas, terem causas multifatoriais, alternam períodos de remissão e exacerbação, evoluem de forma lenta, prolongada e tendem a ser permanentes, desencadeando lesões celulares irreversíveis, podem causar vários graus de incapacidade e ainda evoluir a óbito (BRASIL, 2013). Este tipo de doença torna-se preocupante pois com a tendência do envelhecimento populacional o número de pessoas afetadas por doenças crônicas tende a subir.

Entre as DCNT uma particularmente tem preocupado as autoridades em saúde: a obesidade. Ela é caracterizada pelo acúmulo de tecido adiposo excessivo capaz de prejudicar a saúde, situação que afeta o indivíduo de forma sistêmica (PNPI, 2014). Outros autores caracterizam ainda a obesidade como uma doença crônica, complexa e multifatorial, associada a fatores genéticos, psicossociais, culturais, políticos, socioeconômicos e biológicos (GLUCKMAN et al., 2011).

Os obesos podem sofrer alterações de diversas ordens: metabólicas, locomotoras, respiratórias, psicológicas, entre outras, além de terem o fator de risco aumentado para as doenças cardiovasculares, as dislipidemias, a diabetes mellitus tipo II (AHIMA,2013). Atualmente, essa patologia é um dos grandes problemas de saúde pública, pois tem aumentado as taxas de morbimortalidade (SCHMIDT et al., 2018). A obesidade e suas complicações podem evoluir lentamente, e suas consequências se apresentarem de diferentes formas nos indivíduos afetados.

Embora vários estudos mostrem a correlação da obesidade com várias comobirdades do sistema circulatório, endócrino e osteomioarticular, estudos recentes evidenciam uma forte correlação da obesidade com 13 tipos diferentes 
de canceres. Estima-se que aproximadamente $50 \%$ dos cânceres em pessoas abaixo dos 65 anos foram correlacionados à obesidade e sobrepeso, inclusive entre as crianças (MASSETI; DIETZ; RICHARDSON, 2017). Todas comobirdades que afetam adultos obesos aplicam-se às crianças e adolescentes nas mesmas condições, acrescidas de situações como problemas psicossociais e bullying (GROSSMAN, et al., 2017).

Segundo OPAS (2014) a obesidade é uma epidemia e tem atingido de forma contundente as crianças. A obesidade infantil tem uma origem complexa e o progresso no combate a patologia nas crianças vem tendo resultados lentos e inconsistentes. Segundo Abarca-Gómez (2017), o número de crianças obesas, na faixa etária de 5 a 19 anos, nos últimos quarenta anos aumentou dez vezes. Saiu-se de 11 milhões de crianças afetadas em 1975 para 124 milhões em 2016. A tendência confirmando-se, em 2022 se terá mais crianças e adolescentes obesos do que desnutridos em todo o mundo.

No Brasil o cenário segue os padrões internacionais. O sobrepeso ou a obesidade atingem cerca de 9,7\% (3,8 milhões) de crianças na primeira infância. Os dados do Sistema de Vigilância Alimentar Nutricional (SISVAN) mostram que todas as regiões do país ultrapassaram os limites aceitáveis (2,3\%), sendo que as regiões Norte e Nordeste são as que tem maiores prevalências, 8,4\% e 8,5\% respectivamente (BRASIL, 2013).

A situação torna-se mais preocupante, pois há o risco de a obesidade ser passada através das gerações, sendo validada a premissa de que "obesidade gera obesidade" (WHO, 2015). Segundo Ornellas et al (2017), pais obesos conduzem a um metabolismo modificado e a obesidade em seus filhos quando estes chegam a idade adulta.

As Diretrizes Brasileiras de Obesidade mostram uma correlação positiva para a obesidade e o sedentarismo, baixo ou alto peso ao nascer, a obesidade dos pais, o desmame precoce, o manejo incorreto de fórmulas lácteas, entre outros fatores (ABESO, 2016). As causas podem ser diversas e nem sempre são de fácil identificação, portanto se faz necessário prevenir a obesidade nas suas mais diversas fontes.

O manejo da obesidade infantil pode ser mais difícil do que na fase adulta, uma vez que a criança não tem entendimento da doença, suas causas, as consequências e a prevenção (GLUCKMAN et al., 2011). A diminuição da ingestão calórica e o aumento da prática de atividades físicas se fazem tão importantes quanto à educação e o acesso a informações a respeito da patologia e a forma de combatê-la e preveni-la.

Para se ter êxito no combate à obesidade infantil, é fundamental a prática de atividades físicas e diminuição do tempo sedentário (OPAS, 2014). Executar exercícios físicos programados e manter-se ativo deve ser um comportamento incentivado e aumentado entre o público infantil. Infelizmente, segundo OPAS (2014) há uma diminuição da prática de atividade físicas e recreativas pelas crianças que são condicionadas pela diminuição da oferta deste tipo de atividade pelas escolas, a falta de infraestrutura e violência urbana e o aumento do tempo de tela e diversão. Comportamento este que propicia uma maior oportunidade para consumo de alimentos inadequados e maior exposição a propaganda deste tipo de alimento.

Desta forma, é importante trabalhar a prevenção da obesidade desde a primeira década de vida (GOES et al., 2015). A adoção pelos pais e escolas de políticas de ensino nutricional e incentivo a prática de atividades físicas, visando desde cedo o interesse, o entendimento e a mudança de hábitos pela criança, visto 
que os hábitos e preferências começam a se firmar na infância (GOES et al., 2015).

Para ter êxito na transmissão de um novo conhecimento é primordial utilizar uma metodologia de ensino eficaz. Um saber alicerçado na compreensão e reflexão leva a uma postura crítica que é capaz gerar ações que transformem a realidade (SCHRAM; CARVALHO, 2015). Usar informações corretas, fazendo com que o conhecimento se torne verdadeiro para o indivíduo, aumentando o nível de cognição diante de uma situação-problema, é o que propõe o estudo da lógica (OLIVEIRA; ROCHA, 2011).

Utilizar o raciocínio lógico no processo de aprendizagem de outros saberes que não a matemática não é algo novo. O matemático e lógico Bertrand Russell, que viveu no século $X X$, reduziu o estudo da linguagem em sua plenitude às estruturas lógicas, tomando a lógica por um tipo de linguagem ideal (LAURO; BLASIO, 2016). É importante desenvolver o raciocínio lógico para que ele possa ser utilizado como instrumento de escolha de informações mais corretas, de forma crítica e criativa (SOUSA et al., 2015).

Piaget (1971) diz que "os jogos são essenciais na vida da criança sendo a atividade lúdica o berço das suas atividades intelectuais, indispensável por isso, à prática educativa". Através dos jogos, o professor e os pais podem explorar situações vividas pelas crianças e direcioná-las partindo de algo prazeroso para o alcance de metas em relação ao seu desenvolvimento.

Pode-se aliar jogos e educação para promover ensino. Aprender jogando, tornando o aprendizado mais prazeroso para a criança. Segundo Whyte; Smyth; Scherf (2015), esse é justamente o objetivo dos chamados serious games (jogo sério, tradução literal). Para esses autores este tipo de jogo é desenvolvido para promover habilidades e conhecimentos que muitas vezes são de difícil assimilação e não é gratificante para o envolvido. Diferente de um jogo que tem por único propósito o entretenimento, o serious game relaciona o objetivo do jogo a vida real do participante.

Neste contexto, o objetivo deste estudo foi avaliar os efeitos de jogos de raciocínio lógico sobre o sedentarismo no combate à obesidade em crianças de 7 a 9 anos.

\section{MATERIAIS E MÉTODOS}

Trata-se de uma pesquisa longitudinal prospectiva, controlada, aplicada, de cunho exploratório com abordagem predominantemente quantitativa.

A pesquisa foi realizada em duas escolas municipais localizadas na cidade de Petrolina, estado de Pernambuco. As escolas participantes foram designadas pela secretária de educação do município, que é o órgão responsável por conceder a anuência da pesquisa. Uma instituição foi o local de intervenção (aplicação dos jogos), Escola Municipal Anésio Leão; e a outra, Escola Municipal Rubem Amorim, o controle, (sem aplicação dos jogos). Esta classificação foi obtida mediante sorteio.

O tamanho da amostra foi determinado utilizando o software G-Power com nível de significância de $(0,05)$, poder estatístico de $(0,95)$, magnitude de efeito de $(0,80)$ e 2 grupos envolvidos (Teste $t$ - intervenção e controle), indicando 70 sujeitos com mínimo de amostra (35 elementos em cada grupo) para realizar os procedimentos experimentais. 
A população deste estudo compreendeu todos os alunos de 7 a 9 anos, de ambos os sexos, que estejam regularmente matriculados nas escolas participantes do estudo, cursando o $2^{\circ}$ e $3^{\circ}$ ano do ensino fundamental 1.

Os critérios de exclusão foram: Recusa da participação por parte dos pais e/ou recusa voluntária na participação por parte das crianças; o não comparecimento em alguma das etapas da pesquisa; não atingir mais de $75 \%$ de presença nas oficinas; crianças que não tiveram a faixa etária estabelecida mesmo que estejam cursando o $2^{\circ}$ ou $3^{\circ}$ ano escolar do fundamental I; apresentar patologia de base comprovada que possa afetar o seu crescimento e desenvolvimento cognitivo;

A condição nutricional da criança não foi considerada como critério de inclusão/exclusão, visto que o objetivo da pesquisa era avaliar os efeitos de jogos de raciocínio lógico sobre o sedentarismo no combate à obesidade em crianças de 7 a 9 anos, seja atuando na prevenção das que não possuem a condição ou tratamento das que possuem.

A realização da pesquisa foi efetuada de acordo com as Diretrizes e Normas de pesquisa em seres Humanos, através da resolução 466/2012 do Conselho Nacional de saúde, que inclui o conteúdo do Termo de Assentimento e Consentimento Livre e Esclarecido - TCLE (BRASIL, 2012).

O Projeto foi submetido para aprovação no Comitê de Ética e Deontologia em Estudos e Pesquisa da Universidade Federal do Vale do São Francisco (CEDEP-UNIVASF) obtendo o seu aval sob o CAAE 64231917.6.0000.5196 e número do parecer 1.998.613.

O questionário utilizado foi o Physical Activity Questionnaire for Older Children (PAQ-C). É um instrumento internacional validado (CROCKER et al., 1997) utilizado para mensurar a prática de atividade física por crianças, validado, traduzido e adaptado para o português no Brasil (GUEDES; GUEDES 2015). É composto por 13 questões do tipo check list, tomando como referência atividades realizadas nos últimos 7 dias.

Cada item é medido em uma escala de 5 pontos, excetuando-se as questões 1 e 13, que são listas. Para essas questões é necessário transformar essa escala, pela divisão total de pontos pelo número de atividades na lista, incluindo as que por ventura venham a ser acrescentadas na categoria "outras". O score final foi obtido pelas médias das questões de 1 a 7,9 e 13.

A pesquisa se deu em três etapas: na adaptação dos jogos de raciocínio lógico disponibilizados pelo code.org para a temática do combate à obesidade infantil; na aplicação dos jogos e na reaplicação do questionário ao término das oficinas. As crianças participaram das oficinas na escola, duas vezes por semana, com a duração de 30 minutos cada encontro. Todo o processo de pesquisa aconteceu nas duas escolas, com exceção da aplicação dos jogos que não foi realizada na escola controle.

\section{ADAPTACÃO DOS JOGOS}

Os jogos utilizados para adaptação são da organização code.org; organização sem fins lucrativos, que tem por objetivo a disseminação do ensino da ciência da computação para alunos em todo o mundo, inclusive buscando o aumento da participação da mulheres e minorias sub-representadas.

Os jogos estão disponíveis no site https://code.org/ com opção em língua portuguesa. Cada jogo ou atividade utilizado na pesquisa é gerado a partir de um plano de aula disponibilizado pelo code.org, que por sua vez é traduzido e posteriormente adaptado para a temática da obesidade infantil. Os planos de 
aula sofreram modificações na estrutura e conteúdo para melhor se adequarem a proposta da pesquisa. Foram divididos em: tema; introdução, vocabulário e objetivos; desenvolvimento da atividade, fixação e referência. Na ilustração 01, um exemplo de plano de aula depois da adaptação.

\section{PLANO DE AULA 01}

\section{TEMA AULA:}

- O que é alimentação saudável?

- Diferença entre alimentos processados e in natura.

AULA 01
- Tempo de aula: 20 minutos:
- Introdução e vocabulário.
- Desenvolvimento da Atividade
- Ficaçăo
- Referências

2. INTRODUÇÃO, VOCABULÁRIO E OBJETIVOS

$\mathrm{Na}$ raiz de toda ciência da computação é algo chamado um algoritmo. A palavra "algoritmo" pode soar como algo complicado, mas na verdade é apenas uma lista de instruções que alguém pode seguir para alcançar um resultado. Para fornecer uma base sólida para o resto do ensino de ciência da computação dos seus alunos, vamos focar na construção de um relacionamento seguro com algoritmos. Os objetivos são: listar etapas para mover o personagem pelo mapa, organizar as rotas para alcançar o objetivo predeterminado, prever onde o personagem vai pousar, dada uma lista de etapas e aprender a diferenciar um alimento in natura de um alimento processado.

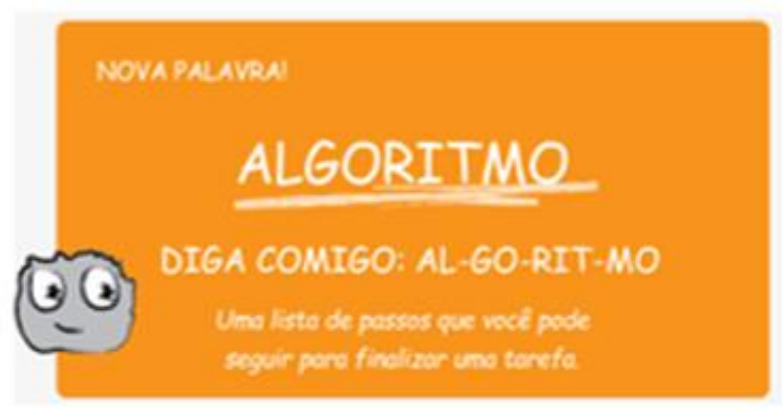


- Algoritmo - Diga comigo: Al-go-rit-mo Uma lista de passos que você pode seguir para concluir uma tarefa.

- Alimentos Saudáveis: "In Natura" - São aqueles obtidos diretamente de plantas ou de animais (como folhas e frutos ou ovos e leite) e adquiridos para consumo sem que tenham sofrido qualquer alteração após deixarem a natureza

- Alimentos Não Saudáveis: "Processados" - Aquele que não pode ser obtido através da natureza, ou seja, é produzido pelo homem através de uma matéria prima conseguida muitas vezes pela natureza. Estes alimentos têm em sua composição produtos artificiais, como corantes, além de terem uma quantidade maior de sal, gordura e açúcar.

\section{DESENVOLVIMENTO DA ATIVIDADE: MAPA DAS FRUTAS}

- Explicar os conceitos dos alimentos

- Mostrar gravuras dos alimentos In Natura e Processados

- Pedir para os alunos dizerem o que é In Natura e Processados de acordo com as figuras mostradas no quadro pelo projetor.

- Depois projetar mapas treino no quadro com figura de alimento in natura e outro processado e com ajuda das setas os alunos precisam chegar até 0 alimento saudável. (Imprimir e entregar as setas em papel ofício A4).

- Explicar o jogo dos Mapas Felizes e seus direcionamentos

- Entregar o pacote de atividades

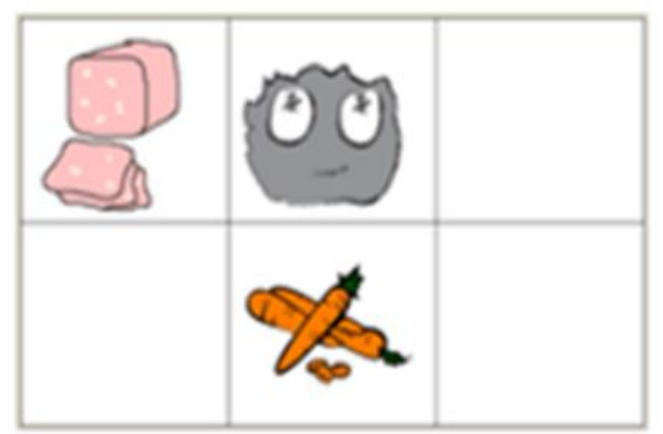

FIGURA 01: Exemplo de mapa treino 


\subsection{Material}

- Pacote de atividades: Planilha mapa felizes, tesoura sem ponta

\subsection{Direcionamentos}

- A planilha dos mapas felizes ajuda a ensinar os alunos a pensar no futuro, a fim de planejar um percurso para o Limus chegue ao alimento in natura e evite os processados.

- Entregar o pacote de atividades

- Um integrante da dupla monta um desafio para que o outro através das setas indiquem passo a passo o caminho correto que o Limus deve seguir para chegar ao alimento adequado.

- Montada a resposta, deve ser mostrada ao professor.

- Se sua resposta for correta, será a vez do outro integrante da dupla montar o desafio.

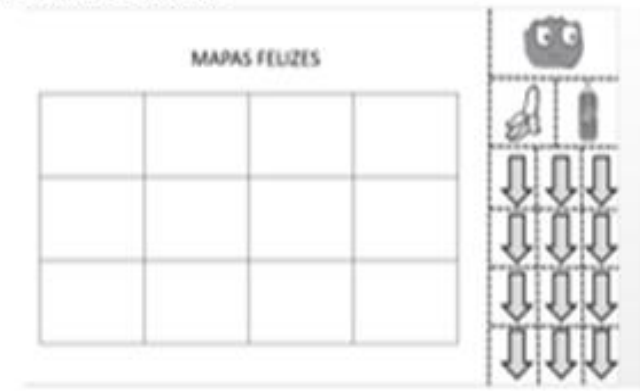

FIGURA 02: Planilha Mapas Felizes

\section{FIXAÇÃO}

\section{$\mathrm{O}$ que aprendemos?}

- Como você se sentiu guiando o Limus para o alimento saudável?

- Você agora consegue escolher seus alimentos saudáveis?

- O que aprendemos hoje? Algoritmo, Alimentos saudáveis e Alimentos Industrializados

\section{REFERẼNCIA}

https://code.org/curriculum/course1/1/Teacher

Fonte: autora (2017)

Os jogos são atividades de Computer ScienceUnplugged (Ciência da Computação Desplugada), cujo a proposta é ensinar ciência da computação sem computador, utilizando jogos com materiais diversos, como cordas, giz de cera, quebra-cabeças, entre outros, além de atividades ao ar livre.

Foram elaborados 12 planos de aulas, extraídos das aulas offline do site do Code.org e adaptados para a temática do combate da obesidade infantil. Cada plano de aula gerou um jogo (com exceção de um dos planos que gerou dois jogos) que foi aplicado em crianças de 7 a 9 anos do ensino fundamental I. 
Entre os doze jogos foram incluídos conceitos de lógica de programação e o estimulo para a mudança de comportamento em relação aos hábitos alimentares e a prática de educação física como forma de combater a obesidade infantil. Todo conteúdo foi exposto somente através das instruções e prática dos jogos. Os conceitos repetiram-se ao decorrer dos 12 jogos para maior fixação. 0 quadro 01 mostra a divisão do conteúdo programático por plano de aula disponibilizado no code.org e seu respectivo endereço eletrônico.

Quadro 01: Conteúdo programático por plano de aula e endereço eletrônico

\begin{tabular}{|c|c|c|c|}
\hline $\begin{array}{l}\text { Número do } \\
\text { plano de } \\
\text { aula } \\
\text { adaptado }\end{array}$ & $\begin{array}{c}\text { Conceito aplicado } \\
\text { no combate a } \\
\text { obesidade infantil }\end{array}$ & $\begin{array}{l}\text { Conceito } \\
\text { aplicado de } \\
\text { lógica de } \\
\text { programaçäo }\end{array}$ & $\begin{array}{c}\text { Endereço eletrónico do plano de } \\
\text { aula original disponibilizado no } \\
\text { code.org }\end{array}$ \\
\hline PLANO 1: & $\begin{array}{l}\text { Hábitos de } \\
\text { alimentaçăo } \\
\text { saudável }\end{array}$ & Algoritmo & $\begin{array}{l}\text { https://code.org/curriculum/course } 1 / 1 / T \\
\text { eacher }\end{array}$ \\
\hline PLANO 2: & $\begin{array}{l}\text { Importância da } \\
\text { prática de } \\
\text { atividades física } \\
\end{array}$ & Programa & $\begin{array}{l}\text { https://code.org/curriculum/course } 1 / 2 / T \\
\text { eacher }\end{array}$ \\
\hline PLANO 3 & $\begin{array}{l}\text { Hábitos de } \\
\text { alimentaçăo } \\
\text { saudável } \\
\end{array}$ & Algoritmo & $\begin{array}{l}\text { https://code.org/curriculum/course } 1 / 6 / T \\
\text { eacher }\end{array}$ \\
\hline PLANO 4 & $\begin{array}{l}\text { Importância da } \\
\text { prática de } \\
\text { atividades física }\end{array}$ & Laço de repetiçäo & $\begin{array}{l}\text { https://code.org/curriculum/course 1/12/ } \\
\text { Teacher }\end{array}$ \\
\hline PLANO 5 & $\begin{array}{l}\text { Importância da } \\
\text { prática de } \\
\text { atividades física }\end{array}$ & Evento & $\begin{array}{l}\text { https://code.org/curriculum/course 1/15/ } \\
\text { Teacher }\end{array}$ \\
\hline PLANO6 & $\begin{array}{l}\text { Importância da } \\
\text { prática de } \\
\text { atividades física } \\
\end{array}$ & Evento & $\begin{array}{l}\text { https://code.org/curriculum/course } 1 / 15 / \\
\text { Teacher }\end{array}$ \\
\hline PLANO 7 & $\begin{array}{l}\text { Obesidade: } \\
\text { conceito, causas e } \\
\text { consequências } \\
\end{array}$ & $\begin{array}{l}\text { Algoritmo } \\
\text { Programa }\end{array}$ & $\begin{array}{l}\text { https://code.org/curriculum/course } 2 / 1 / T \\
\text { eacher }\end{array}$ \\
\hline PLANO 8 & $\begin{array}{l}\text { Obesidade: } \\
\text { conceito, causas e } \\
\text { consequèncias } \\
\end{array}$ & Algoritmo & $\begin{array}{l}\text { https://code.org/curriculum/course } 2 / 2 / T \\
\text { eacher }\end{array}$ \\
\hline PLANO 9 & $\begin{array}{l}\text { Importância da } \\
\text { prática de } \\
\text { atividades física } \\
\end{array}$ & Laço de repetiçäo & $\begin{array}{l}\text { https://code.org/curriculum/course2/5/T } \\
\text { eacher }\end{array}$ \\
\hline PLANO 10 & $\begin{array}{l}\text { Hábitos de } \\
\text { alimentaçăo } \\
\text { saudável } \\
\end{array}$ & Condicionais & $\begin{array}{l}\text { https://code.org/curriculum/course2/12/ } \\
\text { Teacher }\end{array}$ \\
\hline PLANO 11 & $\begin{array}{l}\text { Obesidade: } \\
\text { conceito, causas e } \\
\text { consequências } \\
\end{array}$ & Binário & $\begin{array}{l}\text { https://code.org/curriculum/course2/14/ } \\
\text { Teacher }\end{array}$ \\
\hline PLANO 12 & $\begin{array}{l}\text { Importância da } \\
\text { prática de } \\
\text { atividades física } \\
\end{array}$ & Evento & $\begin{array}{l}\text { https://code.org/curriculum/course2/15/ } \\
\text { Teacher }\end{array}$ \\
\hline
\end{tabular}

FONTE: a autora (2017). 


\section{ANÁLISE DOS DADOS E TRATAMENTO ESTATÍSTICO}

Os dados foram tabulados e armazenados em banco desenvolvido no programa Microsoft Office Excel. Todos os dados analisados utilizando o software estatístico Statistical Package for the Social Sciences (SPSS, versão 20.0) for Windows, com nível de significância estipulado em $p \leq 0,05$ para todas as análises.

Para a caracterização dos participantes do estudo foi empregada à estatística descritiva (média e desvio-padrão). Nas análises que envolveram os 2 grupos (com e sem intervenção) foram utilizados teste t independente para comparar as médias das variáveis estudadas.

Para testar a normalidade dos dados foi aplicado teste de Shapiro Wilk. As variáveis que não apresentaram normalidade serão normalizadas por meio da transformação Box Cox e de um valor lambda estimado.

Primeiramente foi realizado um teste t pareado comparando valores iniciais e finais dos escolares para todas as variáveis do estudo. Em seguida, análise de variância (ANOVA one-way) foi utilizada para comparar as variáveis independentes (antropométricas, QV) com 4 grupos (Pré e Pós simultaneamente): grupo 1: controle - 7 anos; grupo 2: intervenção - 7 anos; grupo 3: controle -8 anos; grupo 4: intervenção - 8 anos. Cada condição da ANOVA foi seguida por teste de comparação de médias Bonferroni, para determinar onde as diferenças significativas ocorreram.

Para determinar as relações entre AF com indicadores de obesidade foi realizado correlação de Pearson e correlações parciais controladas por sexo (caso necessário).

\section{RESULTADOS E DISCUSSÃO}

A amostra deste estudo foi composta de estudantes de duas escolas distintas, uma escola caso, outro controle. Na escola caso, a amostra total era de 65 voluntários, e no controle 55 voluntários. No entanto, cinco crianças enquadravam-se no critério de exclusão: "Apresentar patologia de base comprovada que possa afetar o seu crescimento e desenvolvimento cognitivo"; uma na escola caso e quatro no controle, totalizando 115 participantes na pesquisa. Dos 115 participantes, 56,52\% eram do sexo masculino e 43,48\% eram do sexo feminino.

Vale salientar que para cada variável analisada o valor de " $n$ " pode sofrer variação, visto que a coleta não foi realizada em um único dia, portanto, houve variabilidade no número da mostra, desta forma o valor de "n" é indicado em cada tabela.

Conforme instruções para cálculo do escore contida no instrumento, analisamos três médias. No cálculo da média 1 foram utilizadas as questões de 1 a 7 que mensuram a frequência de atividade física nos últimos sete dias. A média 2 foi obtida pela questão 9 que avalia a frequência de atividade física nos últimos sete dias no tempo livre. A média final foi composta pelas questões 1 a 7; 9 e 13 e a média de TV pela questão 8 que mensurava a quantidade de horas assistindo televisão. 
Tabela 01 - Características descritivas do nível de atividade física da amostra

\begin{tabular}{|c|c|c|c|c|c|c|c|c|}
\hline & $\begin{array}{c}\text { Amostra } \\
\text { Controle } \\
\text { Pré (n) }\end{array}$ & $\begin{array}{l}\text { Amostra } \\
\text { Controle } \\
\text { Pós (n) }\end{array}$ & $\begin{array}{c}\text { Amostra } \\
\text { Intervençăo } \\
\text { Pré (n) }\end{array}$ & $\begin{array}{c}\text { Amostra } \\
\text { Intervençăo } \\
\text { Pós (n) }\end{array}$ & $\begin{array}{c}\text { GRUPO } \\
01 \\
\text { Controle } \\
\text { Pré }\end{array}$ & $\begin{array}{c}\text { GRUPO } \\
02 \\
\text { Controle } \\
\text { Pós }\end{array}$ & $\begin{array}{c}\text { GRUPO } \\
03 \\
\text { Interven- } \\
\text { cão Pré }\end{array}$ & $\begin{array}{c}\text { GRUPO } \\
04 \\
\text { Interven- } \\
\text { ção Pós }\end{array}$ \\
\hline $\begin{array}{l}\text { Variáveis } \\
\text { Subjetivas }\end{array}$ & & & & & $\begin{array}{l}\text { Média } \\
( \pm) D P\end{array}$ & $\begin{array}{l}\text { Média } \\
( \pm) D P\end{array}$ & $\begin{array}{l}\text { Média } \\
( \pm) D P\end{array}$ & $\begin{array}{l}\text { Média } \\
( \pm) D P\end{array}$ \\
\hline $\begin{array}{l}\text { MEDIA } 1 \\
\text { (Questôes } \\
\text { 1 a 7): }\end{array}$ & 30 & 30 & 30 & 30 & $\begin{array}{c}2,97 \pm 0,4 \\
1\end{array}$ & $\begin{array}{c}2,16 \pm 0,6 \\
7\end{array}$ & $\begin{array}{c}2,64 \pm 0,7 \\
2\end{array}$ & $\begin{array}{c}2,77 \pm 0,8 \\
8\end{array}$ \\
\hline $\begin{array}{l}\text { MEDIA } 2 \\
\text { (Questäo } \\
\text { 9): }\end{array}$ & 29 & 30 & 30 & 30 & $\begin{array}{c}1,96 \pm 1,2 \\
0\end{array}$ & $\begin{array}{c}1,66 \pm 1,1 \\
2\end{array}$ & $2 \pm 1,41$ & $\begin{array}{c}2,46 \pm 1,5 \\
4\end{array}$ \\
\hline $\begin{array}{l}\text { MEDIA } 3 \\
\text { (Questão } \\
\text { 13): }\end{array}$ & 30 & 30 & 30 & 30 & $\begin{array}{c}3,47 \pm 1,0 \\
3\end{array}$ & $\begin{array}{c}2,70 \pm 1,3 \\
5\end{array}$ & $\begin{array}{c}3,12 \pm 1,2 \\
9\end{array}$ & $\begin{array}{c}2,72 \pm 1,3 \\
7\end{array}$ \\
\hline $\begin{array}{c}\text { MEDIA } \\
\text { FINAL } \\
\text { (Questôes } \\
\text { de } 1 \text { a } 7 ; 9 \\
\text { e 13): }\end{array}$ & 30 & 30 & 30 & 30 & $\begin{array}{c}2,80 \pm 0,5 \\
7\end{array}$ & $\begin{array}{c}2,17 \pm 0,7 \\
7\end{array}$ & $\begin{array}{c}2,57 \pm 0,8 \\
7\end{array}$ & $\begin{array}{c}2,64 \pm 0,8 \\
9\end{array}$ \\
\hline $\begin{array}{l}\text { MEDIA de } \\
\text { TV }\end{array}$ & 30 & 17 & 30 & 30 & $\begin{array}{c}3,63 \pm 2,9 \\
1\end{array}$ & $\begin{array}{c}5,05 \pm 2,5 \\
3\end{array}$ & $5,2 \pm 4,69$ & $4,5 \pm 3,80$ \\
\hline
\end{tabular}

TABELA 02 - Distribuição das variações do nível de atividade física da amostra

\begin{tabular}{|c|c|c|c|c|c|}
\hline & $\begin{array}{c}\text { GRUPO } 01 \\
\text { Controle Pré }\end{array}$ & $\begin{array}{c}\text { GRUPO 02 } \\
\text { Controle Pós }\end{array}$ & $\begin{array}{c}\text { GRUPO 03 } \\
\text { Intervenção } \\
\text { Pré }\end{array}$ & $\begin{array}{c}\text { GRUPO 04 } \\
\text { Intervençãa } \\
\text { Pós }\end{array}$ & $\begin{array}{c}\text { ANOVA } \\
\text { ONE WAY }\end{array}$ \\
\hline \multicolumn{6}{|l|}{$\begin{array}{l}\text { Variáveis } \\
\text { Subjetivas }\end{array}$} \\
\hline & Média $( \pm)$ DP & Média $( \pm)$ DP & Média ( $( \pm)$ DP & Média $( \pm) D P$ & \\
\hline $\begin{array}{c}\text { MEDIA 1 } \\
\text { (Questões 1 a } \\
7 \text { ) } \\
\text { MEDIA 2 } \\
\text { (Questão 9) }\end{array}$ & $2,97 \pm 0,41^{2}$ & $2,16 \pm 0,67^{1,4}$ & $2,64 \pm 0,72$ & $2,77 \pm 0,88^{2}$ & $0,0003^{* \star}$ \\
\hline $\begin{array}{c}\text { MEDIA } 3 \\
\text { (Questão 13) }\end{array}$ & $3,47 \pm 1,03$ & $2,70 \pm 1,35$ & $3,12 \pm 1,29$ & $2,72 \pm 1,37$ & 0,063 \\
\hline $\begin{array}{l}\text { MEDIA FINAL } \\
\text { (Questões de } \\
\text { 1 a 7; } 9 \text { e 13): }\end{array}$ & $2,80 \pm 0,57^{2}$ & $2,17 \pm 0,77^{1}$ & $2,57 \pm 0,87$ & $2,64 \pm 0,89$ & $0,0197^{*}$ \\
\hline MEDIA de TV & $3,63 \pm 2,91$ & $5,05 \pm 2,53$ & $5,2 \pm 4,69$ & $4,5 \pm 3,80$ & 0,3823 \\
\hline
\end{tabular}

FONTE: a autora (2018)

1 Diferença significativa (Anova one-way, pos hoc Bonferroni) com grupo 1.

2 Diferença significativa (Anova one-way, pos hoc Bonferroni) com grupos 2.

${ }^{4}$ Diferença significativa (Anova one-way, pos hoc Bonferroni) com grupo 4

$\mathrm{Na}$ tabela 02, observa-se que o grupo 2 (controle) manteve-se mais sedentário após a intervenção, ao passo que o grupo 04 (intervenção) tornou-se mais ativo que o grupo controle após a intervenção. 
Na média final o grupo controle pós-intervenção tornou-se mais sedentário do que o controle pré-exposição. Enquanto o grupo intervenção não mostrou resultados estatisticamente significativos. Os resultados sugerem que se nenhuma intervenção for realizada a tendência é que as crianças se tornem cada vez mais sedentárias, comportamento este que é um fator de risco para desenvolvimento da obesidade. No fator sedentarismo, o fato das crianças do grupo caso não alterarem significativamente seus índices pode ser um resultado positivo da intervenção.

Nas demais médias não houve alterações significativas.

No quadro 02, uma representação gráfica do nível de atividade física da Média Final dos quatro grupos, obtidos através dos scores do questionário $P A Q-C$ classificadas de acordo com a sintaxe abaixo:

\section{QUADRO 02 - Classificaçäo do nivel de atividade física por score obtido segundo Olivera et al.,} (2009)

\begin{tabular}{|c|c|}
\hline CLASSFICACAO & SCORE \\
\hline Muito sedentário & Até 1,99 \\
\hline Sedentário & 2 atte 2,99 \\
\hline Moderadamente Ativo & 3 atte 3,99 \\
\hline Ativo & 425 \\
\hline
\end{tabular}

ILUSTRAÇÄO 02 - Nível de atividade física da Média Final por grupo

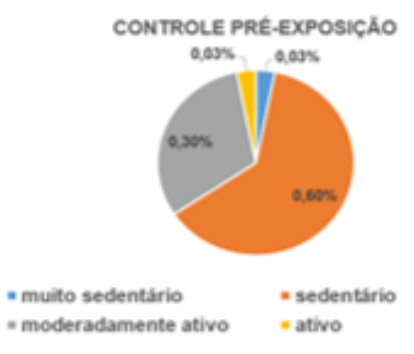

INTERVENÇĀO PRE.EXPOSIÇĀO

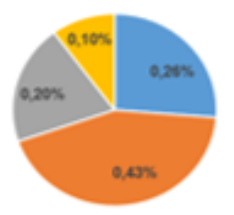

muito sedentario

- moderadamente ative ativo

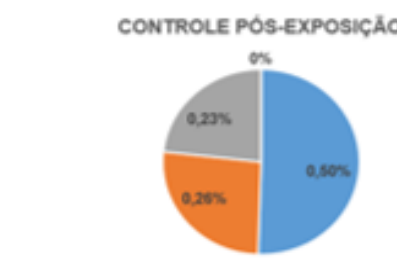

- muito sedentario moderadamente ative

\section{INTERVENÇĀO PÖS-EXPOSIÇĀo}

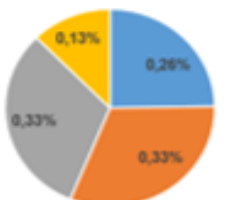

- muito sedentário

- moderadamente ativo
- sedentirio

" ativo

FONTE: a autora (2018).

Com base nos gráficos se pode observar que no grupo controle há um aumento considerável na porcentagem de crianças muito sedentárias passando de 0,03\% 
para $0,50 \%$ acompanhada de uma redução na porcentagem de crianças moderadamente ativas $0,30 \%$ para $0,23 \%$ e a extinção de crianças ativas.

Segundo FERRARI et al. (2018) o tempo sedentário é entendido como atitudes que demandam pouco gasto de energia e acontecem na posição deitada ou sentada. Muitas atividades cotidianas reforçam o comportamento sedentário como estudar, assistir TV e a locomoção motorizada. Crianças devem diminuir o tempo sedentário e aumentar o tempo dispendido em atividades que proporcionem gasto energético para prevenção à obesidade.

Já no grupo intervenção as porcentagens não sofreram alterações tão bruscas, diminuindo o sedentarismo e aumentando a porcentagem de crianças ativas e moderadamente ativas o que pode ser um resultado positivo da intervenção.

A consciência sobre os números da obesidade e sobrepeso na infância e suas consequências mobilizaram diversos órgãos de saúde no mundo e geraram diversos planos de ação. Em todos eles há ao menos um ponto comum: a importância da prática de atividade física no controle da obesidade infantil (ONIS, 2015).

As Diretrizes clínicas para tratamento da obesidade de países como Reino Unido, Austrália e Canadá recomendam uma combinação práticas para combate a obesidade infantil e entre elas a prática de atividade física por mais de 60 minutos por dia de intensidade moderada ou vigorosa e a redução do comportamento sedentário, como por exemplo tempo de tela menor que duas horas (MARTIN et al., 2018).

A intervenção trabalhou também, com intuito de empoderar estas crianças com informações a respeitos de bons hábitos alimentares e incentivando a prática de exercícios físicos utilizando para isso jogos desplugados de lógica de programação. O grupo intervenção pós-exposição mostrou-se menos sedentário do que o grupo controle pós-exposição o que pode ter sido um efeito positivo da pesquisa. Todos os grupos tiveram um tempo médio de tela maior do que duas horas, apontando para um comportamento sedentário que predispõe a obesidade.

A prática de atividade física ou comportamento sedentário pode ter uma influência determinante na perda de peso, sugere-se que o comportamento sedentário contribua para o aumento do peso (DALY; FOOTE e WADSWORTH, 2017). Oferecer as crianças atividades ao ar livre, meios de transportes ativos e atividades recreativas como alguns dos jogos propostos na intervenção, são alternativas indicadas para aumento nos níveis de atividade física das crianças (DALY; FOOTE e WADSWORTH, 2017).

Um ponto a ser considerado é que a grande maioria das crianças frequentam a escola e lá passam considerável parte do seu tempo, e neste local são formadas e informadas sobre diversas questões, é de extrema importância que a escola seja um local de promoção de hábitos saudáveis e promotora de prática de incentivos à atividade física. Segundo Elvasaas et al., (2017) a escola é um ambiente propício para intervenções no combate à obesidade infantil, visto que, muitas crianças não têm recursos, oportunidades e boa educação e apoio fora de seus lares.

Segundo Santos (2016), as aulas de educação física não suprem o tempo suficiente para satisfazer a necessidade de atividade física que é necessária à uma criança semanalmente, porém, estas aulas têm o papel de despertar e orientar as crianças à formação do hábito da prática regular de exercícios físicos tão importantes para prevenção e tratamento da obesidade.

A literatura não entra em consenso sobre qual ser o tempo mínimo ideal para se obter resultados antropométricos e comportamentais mais coesos para combate 
a obesidade infantil. A tendência Segundo Guerra, Silveira; Salvador (2016) é de que seis meses de intervenção é o tempo mínimo para se obter resultados mais favoráveis para formação de hábitos de atividade física e superiores a isto para alterações comportamentais e que quanto maior o tempo de intervenção, maiores serão as mudanças nestas variáveis.

\section{CONSIDERAÇÕES FINAIS}

A prática de atividades físicas das crianças aumentou após a intervenção no grupo exposto aos jogos, elas tornaram-se mais moderadamente ativas. Já no grupo controle elas tornaram-se mais sedentárias ao invés de manterem os mesmos índices de antes da intervenção como previsto. Este resultado mostra que a intervenção pode ter sido eficaz em estimular a prática de atividade física, bem como, se nenhuma medida de intervenção ocorrer no sentido de combate a obesidade as crianças tendem a aumentar o comportamento sedentário.

Este estudo teve limitações, entre elas: o tempo de intervenção, três meses, que foi curto para se obter resultados mais sólidos como indica a literatura; a possível dificuldade de leitura e compreensão do questionário por parte das crianças, já que era auto administrável, pois apesar da maioria já ser alfabetizada muitas demonstraram dificuldade na leitura e compreensão no momento do preenchimento do questionário e a disponibilidade de dias e horários ofertados pelas escolas. Algumas vezes por conta de atividades escolares não previstas ou não comunicadas, as oficinas tiveram que ser adiadas por até duas semanas, causando quebra de continuidade no processo de avaliação.

Os resultados revelam a necessidade de intervenções de combate a obesidade infantil para a promoção de bons hábitos alimentares e práticas de atividade física. A escola é um lugar estratégico para este tipo intervenção que busca mudanças comportamentais e muitas vezes é pouco explorada neste sentido. A pesquisa terá continuidade com estudos follow-up: estudo de impacto após 6 meses de intervenção e um estudo de continuidade com o mesmos objetivo e amostra deste estudo.

\section{REFERÊNCIAS}

ABARCA-GÓMEZ, L. et al. Worldwide trends in body-mass index, underweight, overweight, and obesity from 1975 to 2016: a pooled analysis of 2416 population-based measurement studies in 128. 9 million children, adolescents, and adults. The Lancet, v. 390, n. 10113, p. 2627-2642, dez. 2017.

AHIMA, R. S.; LAZAR, M. A. The health risk of obesity-better metrics imperative. Science, v. 341, n. 6148, p. 856-858, ago. 2013.

ASSOCIAÇÃO BRASILEIRA PARA O ESTUDO DA OBESIDADE E DA SÍNDROME METABÓLICA (ABESO). Diretrizes brasileiras de obesidade 2016 / ABESO - Associação Brasileira para o Estudo da Obesidade e da Síndrome Metabólica. - 4.ed. - São Paulo, 2016. Disponível em: <http://www.abeso.org.br/uploads/downloads/92/57fccc403e5da.pdf>. Acesso em: jan 2017.

BRASIL, C. N. S. Resolução 466/2012-Normas para pesquisa envolvendo seres humanos. Brasília, DF, 2012. 
BRASIL. Ministério da Saúde. Secretaria de Atenção à Saúde. Departamento de Atenção Básica. Diretrizes para o cuidado das pessoas com doenças crônicas nas redes de atenção à saúde e nas linhas de cuidado prioritárias. Brasília : Ministério da Saúde, 2013. 28 p. : il.

CROCKER, P. R. et al. Measuring general levels of physical activity: preliminary evidence for the Physical Activity Questionnaire for Older Children. Medicine and science in sports and exercise, v. 29, n. 10, p. 1344-1349, out. 1997.

DALY, C. M.; FOOTE, S.J.; WADSWORTH, D. D. Physical Activity, Sedentary Behavior, Fruit and Vegetable Consumption and Access: What Influences Obesity in Rural Children?. Journal of community health, v. 42, n. 5, p. 968973, abr. 2017.

ELVSAAS, I. K. et al. Multicomponent Lifestyle Interventions for Treating Overweight and Obesity in Children and Adolescents: A Systematic Review and Meta-Analyses. Journal of Obesity, Article ID 5021902, 14 pages. https://doi.org/10.1155/2017/5021902, 2017

FERRARI, G. L.M. et al. Factors associated with objectively measured total sedentary time and screen time in children aged 9-11 years. Jornal de pediatria,Rio de Janiero, p.1-12, 2018

GLUCKMAN, P. D.; HANSON, M.; ZIMMET, P.; FORRESTER, T. Losing the war against obesity: the need for a developmental perspective. Science translational medicine, v. 3, n. 93, p. 93cm19-93cm19, jul. 2011.

GOES, A. R. et al. «Papa Bem»: investir na literacia em saúde para a prevenção da obesidade infantil. Revista portuguesa de saúde pública, v. 33, n. 1, p. 12-23, jan./jun. 2015.

GROSSMAN, D. C. et al. Screening for obesity in children and adolescents: US Preventive Services Task Force recommendation statement. Jama, v. 317, n. 23, p. 2417-2426, jun. 2017

GUEDES, D.P.; GUEDES, J.E. R.P. Medida da Atividade Física em Jovens Brasileiros: Reprodutibilidade e Validade do PAQ-C e do PAQ-A. Revista Brasileira de Medicina do Esporte, v. 21, n. 6, p. 425-432, dez. 2015.

GUERRA, P.H.; SILVEIRA, J.A.C.; SALVADOR, E.P.. Physical activity and nutrition education at the school environment aimed at preventing childhood obesity: evidence from systematic reviews. Jornal de pediatria, v. 92, n. 1, p. 15-23, jan./fev. 2016

LAURO, M.M.; BLASIO, C. Lógica Moderna e Ciências Cognitivas Uma controversa contribuição da lógica no desenvolvimento das ciências da mente. Revista Científica Faminas (Minas Gerais), v.3, n 1, p. 101-110, 2016.

MASSETTI, G. M.; DIETZ, W. H.; RICHARDSON, L. C.Excessive Weight Gain, Obesity, and Cancer: Opportunities for Clinical Intervention. Jama, v. 318, n. 20, p. 1975-1976, nov. 2017. 
MARTIN, A. et al. Physical activity, diet and other behavioural interventions for improving cognition and school achievement in children and adolescents with obesity or overweight. The Cochrane Library, 2018.

OLIVEIRA, P.A.; ROCHA, A.J.O. Raciocínio lógico, conceitos e estabelecimento de Parâmetros para a aprendizagem matemática. Disponível em:

http://www.facitec.br/revistamat/download/artigos/poliana_alves_de_oliveira_ra ciocinio_logico,_conceitos_e_estabelecimento.pdf. Acesso em: 7 nov 2015

ONIS, M. Preventing childhood overweight and obesity. Jornal de pediatria, v. 91, n. 2, p. 105-107, mar./abr. 2015.

ORNELLAS, F.; et al.. Pais obesos levam a metabolismo alterado e obesidade em seus filhos na idade adulta: revisão de estudos experimentais e humanos. Jornal de Pediatria, v. 93, n. 6, p. 551-559, nov./dez. 2017.

PAN AMERICAN HEALTH ORGANIZATION (OPAS). Plan of Action for the Prevention of Obesity in Children and Adolescents. 66th Session of the Regional Committee of WHO for the Americas, 2014.

PIAGET, J.; ÅLVARO CABRAL; OITICICA, C.M. A formação do símbolo na criança: imitação, jogo e sonho, imagem e representação. 1971.

PLANO NACIONAL DA PRIMEIRA INFÂNCIA (PNPI) - Projeto observatório nacional da primeira infância: Mapeamento da Ação Finalística "Criança com Saúde" - Obesidade Infantil, 2014. Disponível em:

http://primeirainfancia.org.br/wp-content/uploads/2015/01/Obesidade-Infantilv6.pdf. Acesso em: out 2015.

SANTOS, C.N. et al. Título: ESCOLA ATIVA-Atividade Física e Saúde em Contexto Escolar. 2016.

SCHMIDT, Leucinéia et al. OBESIDADE E SUA RELAÇÃO COM A MICROBIOTA INTESTINAL. Revista Interdisciplinar de Estudos em Saúde, v. 6, n. 2, 2018.

SCHRAM, S. C.; CARVALHO, M. A. B. O pensar educação em Paulo Freire. 2015. Disponível em:

<http://www.diaadiaeducacao.pr.gov.br/portals/pde/arquivos/852-2.pdf>. Acesso em: jan 2017.

SOUSA, A. et al. O Universo Lúdico da Programação de Computadores com Logo no Ensino Fundamental. In: Anais dos Workshops do Congresso Brasileiro de Informática na Educação. 2015.

WHYTE, E. M.; SMYTH, J. M.; SCHERF, K. S. Designing serious game interventions for individuals with autism. Journal of autism and developmental disorders, v. 45, n. 12, p. 3820-3831, dez. 2015. 


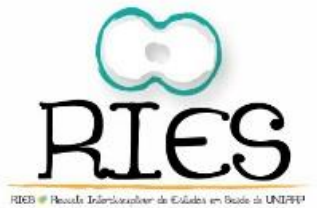

WORD HEALTH ORGANIZATION (WHO). Draft Final Report of the Commission on Ending Childhood Obesity. Geneva, 2015. Disponível em: http://www.who.int/end-childhood-obesity/commission-ending-childhoodobesity-draft-final-report-en.pdf. Acesso em: 11 nov. 2015

Recebido em: 10-07-2018

Aceilto em: 04-10-2019 